\title{
Electrical impedance tomography for chest imaging in acute respiratory failure
}

\begin{abstract}
To the Editor:
Electrical impedance tomography (EIT) is a functional imaging method that can continuously monitor respiratory function at the bedside. Different measures can be generated from EIT patient examinations allowing the assessment of ventilation distribution, regional lung volume changes and respiratory mechanics, as well as lung perfusion, stroke volume or regional oxygen uptake. In a clinical setting, EIT has mainly been applied in patients undergoing mechanical ventilation as summarised in a systematic review published in 2016 [1] and the most recent international consensus statement on EIT chest imaging published in 2017 [2]. EIT has been applied: to detect regional lung overinflation, alveolar collapse and tidal recruitment, and air-leak; to assess patients' responses to changes in ventilator settings and mode, different recruitment manoeuvres or suctioning; and for setting optimum positive end-expiratory pressure. The use of EIT in critical care medicine is increasing.
\end{abstract}

We were therefore pleased to note that EIT has been selected as one of five imaging methods (alongside chest radiography, computed tomography, lung ultrasound and positron emission tomography) in a European Respiratory Society (ERS) statement on chest imaging in acute respiratory failure (ARF) [3]. The aim of this report was to "provide physicians with a comprehensive clinical review of chest imaging techniques for the assessment of patients with acute respiratory failure, based on the scientific evidence as identified by systematic searches". The authors of the document searched the literature on each method from inception to September 2018. Therefore, we expected this report to reflect the previous achievements of EIT already described in detail in the two abovementioned comprehensive reviews $[1,2]$ extended by the publications from about three further years. To our surprise, the Results section of the statement contains only three sentences on EIT in one short paragraph with an additional sentence, stating that "based on our criteria selection, no articles have been found on this topic".

The search criteria used for the review are included as supplemental material. Unfortunately, the accepted version of the ERS statement [3] does not provide the search terms for EIT (only for the other four imaging methods). This could explain, but not justify that all EIT articles on the topic are missing. The constraints for correspondence articles preclude us from providing all EIT articles meeting the general search criteria specified in the Methods section of the statement (original studies in articles written in English, adult humans aged $>18$ years, minimum of 10 study participants), since only ten references are permitted. Nonetheless, we would like to give here a few examples of EIT studies carried out in adult patients with disease entities selected by the task force panel: acute respiratory distress syndrome $[4,5]$ and acute lung injury [6], COPD (acutely exacerbated) [7], pneumonia [8], acute heart failure [9] and pleural effusion [10].

Being experts on EIT, we are primarily qualified to judge the quality of information regarding this technique and not on other imaging methods. The presented results on the other methods are larger than on EIT and they are based on a total of 135 studies. However, some uncertainty exists on the quality of the literature search for these methods as well because tables 1-4 in the statement (outlining the studies included in the preparation of the document) list two chest X-ray, five lung ultrasound, 10 computed tomography and one positron emission tomography studies that do not fulfil the criterion of minimum study participants defined in the Methods. It is also not clear whether studies on healthy subjects and patients not suffering from ARF should have been included.

@ERSpublications

Electrical impedance tomography is a functional imaging method that can continuously monitor respiratory function of critically ill and mechanically ventilated patients at the bedside http://bit.ly/ 2Kt1jF5

Cite this article as: Frerichs I, Zhao Z. Electrical impedance tomography for chest imaging in acute respiratory failure. Eur Respir J 2019; 54: 1901497 [https://doi.org/10.1183/13993003.01497-2019]. 
An update on the use of imaging methods in patients with ARF is topical and relevant. A document reliably summarising the current status of knowledge on all available methods in form of a statement article is of considerable value to clinicians and researchers alike. As little as a decade ago such a review would have been limited to chest radiography and the potential of CT. The pace of change, and potential, in this field is exciting. Thus, it is disappointing that important knowledge in the field of chest EIT has not been included.

We would like to finish our correspondence with one positive statement: regarding EIT, we agree with the authors of the task force report that "EIT is gaining a more clinical role after having represented a research tool for many years" and that "EIT will become more frequently used in patients with acute respiratory failure", as written in the Conclusions. But we recommend that readers who are interested in this method should perform their own literature search and refer to other EIT reviews instead of relying solely on the ERS statement [3].

Inéz Frerichss ${ }^{1}$ and Zhanqi Zhao ${ }^{2,3}$

${ }^{1}$ Dept of Anaesthesiology and Intensive Care Medicine, University Medical Centre Schleswig-Holstein, Campus Kiel, Kiel, Germany. ${ }^{2}$ Dept of Biomedical Engineering, Fourth Military Medical University, Xi'an, China. ${ }^{3}$ Institute of Technical Medicine, Furtwangen University, Villingen-Schwenningen, Germany.

Correspondence: Inéz Frerichs, Dept of Anaesthesiology and Intensive Care Medicine, University Medical Centre Schleswig-Holstein, Campus Kiel, Arnold-Heller-Str. 3, 24105 Kiel, Germany. E-mail: frerichs@anaesthesie.uni-kiel.de

Received: 27 July 2019 | Accepted: 30 July 2019

Conflict of interest: I. Frerichs reports grants from European Union's Horizon 2020 Research and Innovation Programme (Project CRADL, grant agreement number 668259 and Project WELMO, grant agreement number 825572), personal fees for lectures and reimbursement of congress and travel costs from Dräger Medical, outside the submitted work. Z. Zhao reports personal fees for consultancy from Dräger Medical, outside the submitted work.

\section{References}

1 Kobylianskii J, Murray A, Brace D, et al. Electrical impedance tomography in adult patients undergoing mechanical ventilation: a systematic review. J Crit Care 2016; 35: 33-50.

2 Frerichs I, Amato MB, van Kaam $\mathrm{AH}$, et al. Chest electrical impedance tomography examination, data analysis, terminology, clinical use and recommendations: consensus statement of the TRanslational EIT developmeNt stuDy group. Thorax 2017; 72: 83-93.

3 Chiumello D, Sferrazza Papa GF, Artigas A, et al. ERS statement on chest imaging in acute respiratory failure. Eur Respir J 2019; 54: 1900435.

4 Yun L, He HW, Moller K, et al. Assessment of lung recruitment by electrical impedance tomography and oxygenation in ARDS patients. Medicine 2016; 95: e3820.

5 Franchineau G, Brechot N, Lebreton G, et al. Bedside contribution of electrical impedance tomography to setting positive end-expiratory pressure for extracorporeal membrane oxygenation-treated patients with severe acute respiratory distress syndrome. Am J Respir Crit Care Med 2017; 196: 447-457.

6 Pulletz S, Kott M, Elke G, et al. Dynamics of regional lung aeration determined by electrical impedance tomography in patients with acute respiratory distress syndrome. Multidiscip Respir Med 2012; 7: 44.

7 Frerichs I, Achtzehn U, Pechmann A, et al. High-frequency oscillatory ventilation in patients with acute exacerbation of chronic obstructive pulmonary disease. J Crit Care 2012; 27: 172-181.

8 Karsten J, Krabbe K, Heinze H, et al. Bedside monitoring of ventilation distribution and alveolar inflammation in community-acquired pneumonia. J Clin Monit Comput 2014; 28: 403-408.

9 Noble TJ, Morice AH, Channer KS, et al. Monitoring patients with left ventricular failure by electrical impedance tomography. Eur J Heart Fail 1999; 1: 379-384.

10 Becher T, Bussmeyer M, Lautenschlager I, et al. Characteristic pattern of pleural effusion in electrical impedance tomography images of critically ill patients. Br J Anaesth 2018; 120: 1219-1228.

Copyright @ERS 2019

From the authors:

We read with interest the letter from Frerichs and Zhao regarding our recently published European Respiratory Society (ERS) statement [1]. Frerichs and Zhao noted that, although in the task force electrical impedance tomography (EIT) was considered among the imaging techniques used in acute respiratory failure, no related articles were reported in the final paper. In the report, task force members supported by two methodologists performed a systematic search of the literature on five lung imaging techniques (i.e. chest radiography, lung ultrasound, computed tomography, position emission tomography and EIT) using the same search terms for all the techniques (refer to tables S1-S4 in the ERS statement); according to these criteria we did not report any articles related to the EIT. This is due to the fact that the 
systematic literature search did not yield results inherent to the application of EIT in acute respiratory failure. In their correspondence, Frerichs and Zhao cited some studies and reviews on EIT. We are aware of the recent systematic review by KoBYLIANSKII et al. [2] and the recommendations by Frerichs et al. [3] on EIT. Yet, the latter were excluded by the a priori choice of including only original investigations. Therefore, in our search, although many articles were found, these were excluded as they did not fulfil all the predefined criteria (e.g. papers on healthy subjects, case series, reviews and papers not focused on acute respiratory failure were excluded).

Regarding the suggestions of Frerichs and Zhao recommending readers perform their own literature search, this is a personal opinion and we recommend adding the recently published ERS statement to the existing knowledge in the field of imaging in acute respiratory failure.

Independently of the specific task force results, we are aware of plenty of physiological and preclinical papers on EIT and we believe that EIT is a useful bedside technique to evaluate both the distribution of the ventilation and lung recruitment/derecruitment.

\section{@ERSpublications}

Is electrical impedance tomography ready for clinical use in acute respiratory failure? http://bit.ly/ 30Gjvlt

Cite this article as: Chiumello D, Sferrazza Papa GF. Electrical impedance tomography for chest imaging in acute respiratory failure. Eur Respir J 2019; 54: 1901657 [https://doi.org/10.1183/13993003.01657-2019].

Davide Chiumello ${ }^{1,2}$ and Giuseppe Francesco Sferrazza Papa ${ }^{2,3}$

${ }^{1}$ SC Anestesia e Rianimazione, Ospedale San Paolo - Polo Universitario, ASST Santi Paolo e Carlo, Milan, Italy. ${ }^{2}$ Dipartimento di Scienze della Salute, Centro ricerca coordinata di Insufficienza respiratoria, Università degli Studi di Milano, Milan, Italy. ${ }^{3}$ Casa di Cura del Policlinico, Dipartimento di Scienze Neuroriabilitative, Milan, Italy.

Correspondence: Davide Chiumello, SC Anestesia e Rianimazione, Ospedale San Paolo - Polo Universitario, ASST Santi Paolo e Carlo, Milan, Italy. E-mail: davide.chiumello@unimi.it

Received: 20 Aug 2019 | Accepted: 21 Aug 2019

Conflict of interest: D. Chiumello has nothing to disclose. G.F. Sferrazza Papa has nothing to disclose.

\section{References}

1 Chiumello D, Sferrazza Papa GF, Artigas A, et al. ERS statement on chest imaging in acute respiratory failure. Eur Respir J 2019; 54: 1900435.

2 Kobylianskii J, Murray A, Brace D, et al. Electrical impedance tomography in adult patients undergoing mechanical ventilation: A systematic review. J Crit Care 2016; 35: 33-50.

3 Frerichs I, Amato $\mathrm{MB}$, van Kaam $\mathrm{AH}$, et al. Chest electrical impedance tomography examination, data analysis, terminology, clinical use and recommendations: consensus statement of the TRanslational EIT developmeNt stuDy group. Thorax 2017; 72: 83-93. 